\title{
Dans une situation d'incompréhension, un processus très ouvert est nécessaire où la dimension locale est clé*
}

\author{
Y. LE BARS
}

(Manuscrit reçu le 19 septembre 2003, accepté le 21 septembre 2003)

RÉSUMÉ Il faut d'abord constater la grande divergence d'appréciation entre les spécialistes et le public sur l'état de la gestion des déchets radioactifs. L'absence de compréhension commune sur ce qui constitue le risque associé aux déchets rend la négociation du risque au sein de la société difficile. Pour avancer vers des solutions qui respectent le très long terme les États concernés ont mis en place des processus, qui ont certains traits en commun. La gestion par étapes - qui va de pair avec la réversibilité permet d'articuler les différents niveaux et les différentes politiques, tout en donnant le temps à l'observation et à l'apprentissage. La clarté du rôle des différents acteurs, comme leur comportement sont les clés de la consolidation de la confiance. Ia dimension locale du processus est essentielle, et plus particulièrement en France, qui est pour l'instant le seul pays du monde à étudier des stockages en dehors des zones déjà familières du nucléaire. Trois garanties doivent être apportées : celle de la compréhension de la sûreté des installations ; celle de l'équité dans le choix des sites, avec des opportunités de développement apportées par les projets ; enfin celle du débat local ouvert. À ces conditions, on peut espérer définir des voies pour la gestion à long terme des déchets radioactifs, dans une logique de développement durable.

ABSTRACT In a situation of incomprehension, a very opened process is necessary where the local dimension is the key.

First and foremost, it is important to note the largely diverging opinions between specialists and the public on the current state of radioactive-waste management. The lack of a common understanding of what may constitute a waste-induced risk complicates any social negotiation pertaining to risk. In order to proceed towards solutions that are respectful of the long term, the countries concerned have implemented procedures that share many features in common. By selecting a stepwise approach - that is fully in line with the reversibility principle - it is possible to structure the different levels and policies, while allowing for ongoing observation and apprenticeship. The clear identification of the role of the different actors, as well as their behaviours, constitute the key elements for reinforcing their confidence. The local dimension of the procedure is essential and, even more in France, since she remains the only country in the world to investigate the feasibility of waste repositories outside areas that are already familiar with nuclear energy. Three guarantees must be provided: understanding the safety of the facilities, applying equity in the site-selection process while integrating development incentives in the

\footnotetext{
* Cer anicle s’appuic sur l'exposé présenté par Y. Le Bars au quatrième congrès natonal de la Société française de radioprotection, à Montpellier. 11-13 juin 2003, session 7 "radioprolection el gestion des déchets radioactils ”.

I Président du Conseil d'administration de l'ANDRA. président dEDRAM. président du Foram for Stakelueldere' Conlidence (OCDE/ AEN/ RWMC).

ANIDRA, 1/7 rue Jcan-Monnet, 92298 Châtenay-Malabry, France.
} 
projects, and ensuring an open local debate. Under those conditions, it is likely that appropriate means will be defined to manage radioactive waste over the long term while respecting the principle of sustainable development.

\section{Introduction}

La gestion des déchets radioactifs est une tache délicate, qui oblige à en considérer à la fois la dimension technique et scientifique, et la dimension politique et sociale. Elle implique de nombreux acteurs, différents niveaux territoriaux (les collectivités territoriales, le gouvernent et le parlement...), et aussi diverses politiques publiques, comme celles de l'énergie, de l'environnement, de la radioprotection ou du développement local. Cette tâche délicate a connu des avancées, mais aussi des difficultés qui ont parfois condıit à des retours en arrière...

La présentation qui suit s'inspire d'expériences en France et dans plusieurs autres pays, et tire parti des échanges au sein d'EDRAM (Association "for Environmentaly safe Disposal of Radio Active Materials", qui regroupe les dirigeants des agences, analogues à l'ANDRA, en charge de la gestion des déchets radioactifs dans le monde) et du Forum for Stakeholders' Confidence mis en place par I'AEN/RWMC.

On commencera par constater le fossé qu'il y a entre l'évaluation sur la qualité de la gestion assurée en France ou en Europe faite par les spécialistes, et la perception qu'en a le public.

En tirant les leçons de l'EDRAM et du FSC, on développera ensuite les éléments qui contribuent à la construction de la confiance dans la gestion des déchets radioactifs : un processus bien établi, par étapes, avec une structure claire des acteurs, et des comportements reflétant de solides valeurs. Mais, selon les pays, il y a différentes histoires et les étapes se déroulent différemment.

Enfin, on s'attachera aux enjeux locaux des démarches de la gestion des déchets radioactifs. Trois garanties doivent être apportées localement : d'abord et en tout premier lieu, celle de la sûreté à court, moyen et à long terme ; ensuite, celle que des opportunités de développement sont associées à toute installation ; enfin, que les projets s'élaborent dans un processus permettant un dialogue ouvert.

\section{Un fossé entre l'appréciation des spécialistes et celle du public}

Nous devons constater la distance qu'il y a entre ce que disent les techniciens sur la gestion des déchets radioactifs et ce qu'en pense l'opinion. Cela a conduit différents pays à des remises en cause douloureuses de leurs programmes. 


\begin{tabular}{|c|c|c|}
\hline Activité Vie & $\begin{array}{l}\text { Vie courte } \\
<30 \text { ans }\end{array}$ & $\begin{array}{l}\text { Vie longue } \\
>30 \text { ans }\end{array}$ \\
\hline $\begin{array}{l}\text { Très faible activité (TFA } \\
\text { démantèlement, et résidus } \\
\text { miniers) }\end{array}$ & \multicolumn{2}{|c|}{$\begin{array}{l}\text { Centre TFA } \\
1 \text { million de } \mathrm{m}^{3}\end{array}$} \\
\hline Faible activité (FA) & \multirow{2}{*}{$\begin{array}{l}\text { Centre de l'Aube } \\
\text { (stockage de surface) } \\
1300000 \mathrm{~m}^{3}\end{array}$} & $\begin{array}{c}200000 \mathrm{~m}^{3} \\
\text { (déchets radifères, graphites) }\end{array}$ \\
\hline Moyenne activité (MA) & & $\begin{array}{c}60000 \mathrm{~m}^{3} \\
\text { (déchets technologiques) }\end{array}$ \\
\hline Haute activité (HA) & \multicolumn{2}{|c|}{$\begin{array}{l}\text { (déchets vitrifiés, combustibles usés) } \\
\text { - Vitrifiés : } 5000 \mathrm{~m}^{3} \quad \text { (CU : } 3500 \text { t) } \\
\text { A l'étude (loi du } 30 \text { décembre 1991) }\end{array}$} \\
\hline
\end{tabular}

Figure 1 - Volume des déchets produits (autour de 2020).

Volume of produced wastes (around 2020).

(i) Une analyse technique montre que des solutions existent. Ainsi, en France, $95 \%$ du volume des déchets produits trouvent une issue définitive en stockage de surface (Centre de l'Aube) et un centre de stockage va, fin 2003, accueillir les déchets de démantèlement (déchets de très faible activité, TFA) ( $c f$. Fig. 1).

Des améliorations nécessaires ont été repérées, en particulier pour les déchets de faible activité et à vie longue (déchets radifères issus de sites pollués historiques, graphites issus des premières centrales graphite/gaz), et pour la reprise d'entreposages anciens.

Enfin, un processus a été lancé pour définir des solutions à la gestion à long terme des déchets HAVL, pour l'instant entreposés en lieu sûr ( $c f$. Fig. 2).

Les impacts sur l'homme de la gestion des déchets sont très faibles, de plusieurs ordres de grandeur en-dessous de ceux reçus naturellement par l'ensemble de la population ou par les travailleurs du nucléaire du fait de leur activité.

(ii) Pourtant, pour $60 \%$ des Français (BVA 2000), les déchets radioactifs ne sont pas gérés correctement, $65 \%$ pensent qu'on ne leur dit pas la vérité, $5 \%$ seulement accepteraient de vivre à côté d'un stockage (contre 19\% qui accepteraient de vivre à côté d'une centrale). Ces positions sont assez uniformément répandues en Europe, mais Suédois et Finlandais ont davantage confiance dans leurs entreprises et institutions (Eurobarometer 2001). 


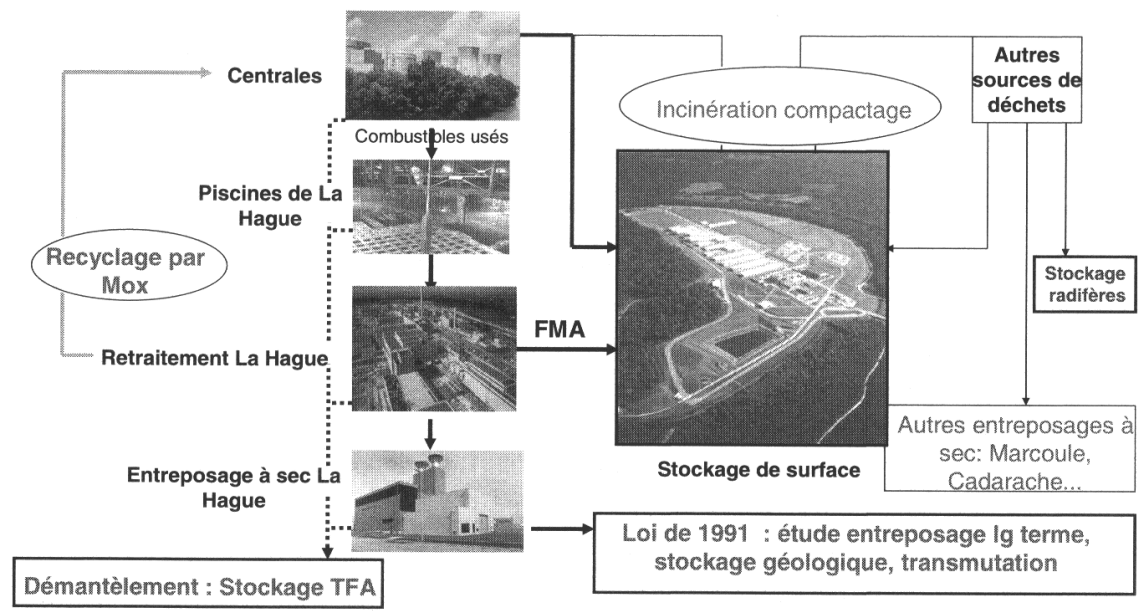

Figure 2 - Le circuit des déchets dans le système français.

The waste way in the French system.

Élément positif : $80 \%$ des Européens disent «la génération qui a utilisé l'électricité est responsable des déchets correspondants ».

Une analyse qualitative (faite par le Credoc à la demande de l'Andra) complète ces données. La gestion des déchets radioactifs n'est pas une préoccupation prioritaire, elle vient derrière les menaces globales, l'insécurité, et même derrière d'autres risques industriels. L'inquiétude diffuse qu'ils provoquent est atténuée quand les gens savent qu'un organisme public en est chargé.

Les déchets radioactifs n'ont pas du tout une bonne image. Si on en fait le portrait par des analogies, on obtient :

- si c'était un insecte : un scorpion,

- si c'était une plante : une plante carnivore,

- si c'était un homme : Hitler, Ben Laden...

Quand on interroge une personne ou un groupe, cela conduit à une attitude $a$ priori de rejet. Il y a un refus du problème, rejet sur les autres, l'industrie, le gouvernement, dans une attitude de «consommateur exigeant non-engagé ».

Il faut avancer dans la discussion pour voir exprimer un sentiment de culpabilité : «c'est vrai que je profite de cette surconsommation qui gaspille et produit des déchets ». 
Ce n'est que plus avant dans la discussion que le citoyen apparaît : « oui, il faut que je m'implique, c'est un problème réel de notre génération (attitude généreuse) et cela pourrait être près de chez moi (NIMBY)».

(iii) Ainsi, il n'y a pas de compréhension commune de ce qui constitue le risque associé aux déchets, de ce que sont la radioactivité et l'impact des faibles doses : il est, de ce fait, difficile de négocier la gestion d'un risque au sein de la société quand il n'y a pas un minimum de consensus sur ce qui provoque ce risque. C'est une tâche très importante pour toute la communauté scientifique, des épidémiologistes, et des radio-protectionnistes en particulier, d'améliorer cette situation : c'est un enjeu considérable, que l'opération menée pour le groupe Radioécologie du Nord-Cotentin a été une des premières à affronter.

À cette tâche, sociologues et psychosociologues méritent d'être associés : le risque radioactif touche à l'espace, la radioactivité le marque durablement, comme l'accident de Tchernobyl le montre encore. Les sites de déchets radioactifs alimentent aussi la crainte d'un marquage sur la longue durée de l'espace par la radioactivité.

Dans plusieurs pays, quand la question n'était abordée que sous l'angle technique, cette attitude de méfiance n'a pas permis aux actions engagées d'aboutir. Le Canada (1998) ou la France (1990, 2000) ont connu ces difficultés. D'autres ont buté de n'avoir pas d'alternative. La Suède, la Finlande, les ÉtatsUnis comme la France ont réussi à mettre en route des démarches positives, à la fois techniques et sociales, qui ont permis des avancées significatives.

\section{Un processus par étapes permet d'avancer}

Il s'agit de remplir une responsabilité de notre génération : réussir une gestion de tous les déchets, et tout particulièrement à très long terme pour ceux de haute activité, alors que la méfiance est la règle à leur sujet.

(i) On doit constater qu'une forme de processus standard s'est progressivement imposée dans le monde pour tenter de dépasser les contradictions constatées : il faut sortir de l'attitude de rejet de la question à celle de citoyen responsable.

Ce processus par étapes, avec des échéances, étudie des alternatives, inclut la définition des sites, mobilise la recherche, associe une évaluation indépendante et fournit des forums de débats pour un apprentissage mutuel entre les différents partenaires. Il donne le pouvoir aux parties qui se sentent concernées d'influencer le projet. Une agence autonome est souvent créée à cette occasion pour mener 
A. Définition du cadre de préparation des
décisions

B. Phase d 'études et de recherches

C. Définition d 'une stratégie, cholx d'une option

D. Sites de stockage éventuel

1. Recherche de sites potentiels

2. Étude de site(s) possible(s) depuis la surface ou en soutertain (laboratoire)

3. Choix d'un site pour un stockage

E. Décision de construction du stockage

F. Exploitation

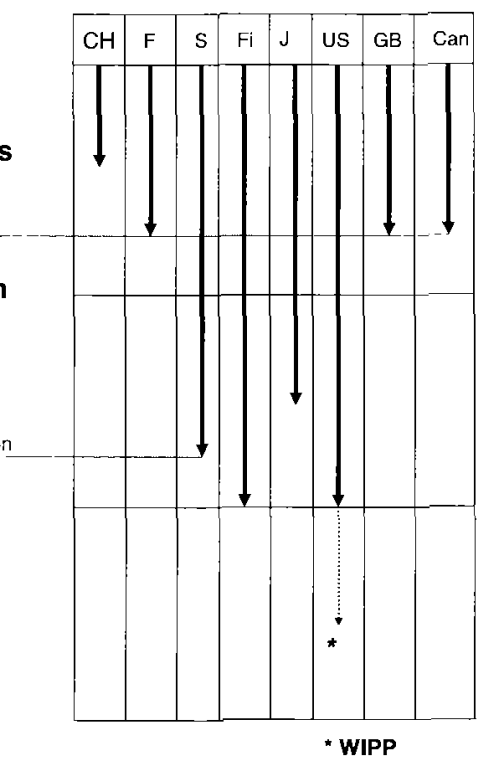

Figure 3 - Situation respective des différents états.

Respective situation in various states.

à bien tout ou partie du programme. La loi française de 1991, celle du Canada en 2000 ont, comme celle du Japon en 2000, l'essentiel de ces caractéristiques.

Regardons comment les programmes se déroulent, selon les différents pays, selon leur propre histoire ( $c f$. Fig. 3).

Certains ont encore à définir ou à préciser le cadre du travail, comme la Suisse. La France et le Canada en sont à une phase de recherche, pour préparer, à la même échéance (2006), des choix de caractère stratégique : le stockage géologique n’est pas retenu a priori comme la solution. C'est ce type de démarche que le gouvernement anglais vient d'adopter pour la Grande-Bretagne.

Pour décider, la France souhaite tirer parti, en 2006, des recherches selon trois axes: l'entreposage de longue durée et les conteneurs de déchets, le stockage géologique profond, la séparation avancée et la transmutation des déchets dans de nouveaux réacteurs. Le Canada compare entreposage sur site des centrales, entreposage centralisé, et stockage géologique profond.

Plusieurs pays ont retenu le stockage géologique comme solution (les ÉtatsUnis, la Suède, la Finlande). Ils sont engagés à différents stades dans la recherche de sites. 
La Finlande expliquera la démarche qui l'a conduite à retenir un site «en principe », c'est-à-dire sous réserve des autorisations à obtenir dans les étapes suivantes. La Suède étudie deux sites candidats.

La France est dans une situation particulière: sans avoir fait le choix du stockage géologique, elle dispose d'un laboratoire profond de caractérisation de site retenu après l'étude de trois sites depuis la surface. Le rapport de l'ANDRA, fin 2005, sur la faisabilité du stockage géologique s'appuiera donc sur l'étude d'un site particulier. La France ne dispose pas de laboratoire méthodologique : elle tire parti de ceux des autres pays (Suisse, Belgique, Canada, Suède).

(ii) La gestion par étapes (ou « adaptive staging », selon la formule retenue aux États-Unis), est maintenant adoptée par tous. Voyons pourquoi, quelles en sont les caractéristiques et comment la réversibilité en tire parti.

Un processus qui implique des dizaines d'années et plusieurs générations, se déroule, par nature même, selon des étapes distinctes. Des instruments comme les directives européennes sur le «Strategical Environmental Assessment » (SEA, au niveau de l'élaboration de la stratégie et des politiques) et «l'Environmental Impact Assessment » (EIA au niveau de l'évaluation du projet) sont déjà une organisation d'étapes clés.

Il s'agit en effet d'articuler différents niveaux (national et local), différentes générations et différentes politiques : la politique de l'énergie, celle de la gestion de tous les déchets, avec ses règles de sûreté et de radioprotection, la politique de recherche de sites, celle de la gestion concrète des projets.

Par exemple, la France vient de relancer un débat national sur les choix énergétiques. On peut penser qu'il conduira à élaborer un plan national de la gestion de tous les déchets qui permettra de préparer le rendez-vous de 2006 en permettant :

- une plus grande convergence entre techniciens et le public sur le diagnostic de la situation actuelle ;

- de repérer les décisions à prendre.

Il pourra s'appuyer sur un nouvel inventaire en cours d'élaboration par l'ANDRA, qui donnera, en 2004, les quantités par types de déchets existants aujourd'hui, et prévus pendant la durée de vie du parc actuel (hypothèse à 40 ans).

La réversibilité est une exigence complémentaire : il s'agit de permettre à la société de revenir sur les décisions antérieures, d'avancer ou de retarder une échéance, de modifier le contenu d'une étape, ceci en fonction de ce qui sera connu 
au moment de la décision. Cela suppose un dispositif d'observation et de recherche pour vérifier que les performances attendues sont bien réalisées.

La France a retenu d'étudier la faisabilité d'un stockage réversible (décision du gouvernement, décembre 1998). C'était en particulier une demande des collectivités territoriales dans la formulation de leur acceptation de l'installation d'un laboratoire souterrain ( $c f$. Annexe 1).

On se trouve ainsi dans des processus dont l'issue ne peut être définie à l'avance : d'autres que les concepteurs du projet auront la capacité d'en orienter le déroulement.

(iii) Avec le Forum for Stakeholders' Confidence, il faut ajouter que le processus ne garantit pas la confiance à lui seul : les rôles respectifs des acteurs et leurs comportements sont essentiels.

Les responsabilités des producteurs de déchets, de l'opérateur, des instances d'évaluation, de l'autorité du contrôle de la sûreté comme des autorités communales et départementales, ainsi que du gouvernement central, doivent être claires, connues et reconnues : cet objectif n'est pas toujours atteint, mais mérite un effort d'amélioration continue. Ainsi, récemment en France, une réforme de l'autorité de sûreté a conduit à regrouper sûreté nucléaire et radioprotection. De plus, un contrat quadriennal a été conclu (et rendu public) entre le gouvernement et chacun des acteurs de la loi de 1991 (ANDRA, CEA). Chaque acteur doit respecter les limites du rôle qui lui est attribué. Un schéma financier pour la prise en charge des dépenses de long terme est en cours d'étude en France.

Le comportement des acteurs doit correspondre aux valeurs de rigueur, d'ouverture au dialogue, en particulier de capacité à écouter. Ce n'est pas toujours facile. Dans ce but, l'ANDRA a, par exemple, adopté une «charte des relations avec ses partenaires $\gg(c f$. Annexe 2$)$.

\section{La dimension locale est déterminante}

Abordons maintenant la dimension locale du processus. C'est une dimension clé du processus sur laquelle plusieurs programmes ont buté : il n'est pas facile d'entrer en discussion localement, alors que l'attitude de rejet a priori domine.

Il est important de remarquer que si les sites des centrales portent des déchets radioactifs, les trois quart du total français compté en becquerels est regroupé à COGEMA/La Hague. Par contre $95 \%$ des volumes vont en stockage de surface au Centre de stockage de l'Aube (cf. Fig. 4). 
- La Hague : 3/4 de la radioactivité en $\mathrm{Bq}$

- 19 sites des centrales $\triangle$ Cadarache et Marcoule : $1 / 4$

- Autres : $<1 \%$, dont le CSA, mais plus de $95 \%$ des volumes

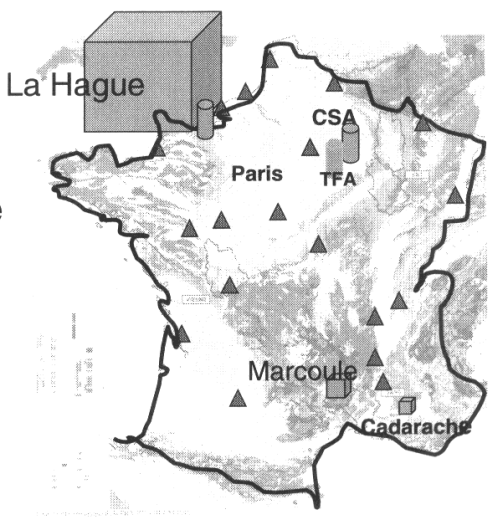

Figure 4 - Localisation des déchets en France. Localisation of waste in France.

Des garanties doivent pouvoir être apportées à la population et aux autorités communales et départementales qui sont concernées par la gestion des déchets. On lira avec intérêt la délibération du syndicat intercommunal à vocation multiple des pays de Saulx et du Perthois du 3 juin 1994, qui accepte que soit étudiée la faisabilité de l'implantation d'un laboratoire souterrain (cf. Annexe 2).

Trois garanties semblent essentielles et concernent la sécurité, le développement local, et le débat.

(i) La première garantie que l'on doit apporter dans la démarche locale, c'est que la sûreté est assurée à court, moyen et long terme.

L'incompréhension des effets de la radioactivité a conduit plusieurs pays à retenir des sites déjà familiers avec le nucléaire civil (Suède, Finlande) ou militaires (États-Unis). La France n'a pas fait ce choix, mais doit alors insister sur la grande qualité de la géologie et ses capacités à retenir les radioéléments à très long terme.

L'existence d'un consensus national sur les moyens d'assurer cette sûreté facilite la définition des sites à étudier ou du site à retenir. Ainsi qu'en est-il de la Suède et de la Finlande avec le concept appelé KBS III, adopté avant toute négociation de site de stockage?

En France, une règle fondamentale de sûreté a défini, dès 1991, des principes généraux à prendre en considération dans différentes conditions géologiques (RFS III.2.f). 
Enfin, un effort de culture scientifique est utile localement pour donner des éléments de la compréhension de la sûreté. Un programme de culture scientifique et technique, non limité aux déchets ou à l'énergie nucléaires, mais couvrant tous les risques (naturels et industriels) est envisagé en Meuse.

(ii) La deuxième garantie est celle de l'équité dans le choix des sites. Cela veut dire en particulier que - à partir du moment où la priorité de la sûreté est reconnue - des opportunités de développement seront offertes aux communes et départements qui acceptent de s'engager dans le processus.

En France, la loi de 1991 a prévu une organisation particulière, pilotée par les départements concernés (les GIP) pour répartir les deux fois 1,5 M€ par an. Autour de Bure, un pôle scientifique et technique est à l'étude pour accueillir des emplois sur des thèmes comme les utilisations des capteurs dans l'environnement, la formation aux travaux ou les utilisations de l'argile.

Il faut aussi savoir prendre en compte les craintes d'effets négatifs. Ainsi, au Canada, une garantie est accordée pour assurer la baisse éventuelle de la valeur des bâtiments dans une commune qui accueille des déchets radioactifs. En France, un contrat du même type avait été préparé pour le vignoble, sur un site de laboratoire, qui n'a finalement pas été retenu (Gard).

On doit constater les impacts économiques positifs des stockages là où ils existent déjà. Le WIPP, qui accueille des déchets militaires, près de Carlsbad (États-Unis), représente $25 \%$ des emplois de la ville et près de $30 \%$ de ses ressources. En France, le Centre de l'Aube a permis à la population de son secteur de croître, alors qu'autour elle a diminué. Le tourisme a pu se développer, avec une rénovation des monuments et de l'habitat.

(iii) Enfin le débat local doit permettre l'expression de tous ceux qui se sentent concernés et qui souhaitent influencer le processus.

Pour cela, l'existence de forum permanent, comme le comité local d'information et de suivi associé au laboratoire souterrain de Bure, en France, peut être utile. Regroupant 90 personnes, élus locaux, associations, experts, et présidé par le préfet du département, il dispose d'un bureau de 20 personnes (qui se réunit tous les mois) et d'un secrétaire scientifique. Il est maître de ses ordres du jour et a des moyens propres ( $2 \mathrm{M} €$ par an) pour la formation de ses membres, les échanges internationaux qu'il décide lui-même et des expertises qu'il mobilise. Il a organisé un colloque sur la « réversibilité et ses limites ». Il participe au réseau européen «COWAM » (Communities Waste Management, concernées par la gestion locale des déchets radioactifs des pays européens), qui regroupe chaque année 120 personnes, $1 / 3$ d'élus, 1/3 de représentants d'associations, et 1/3 venant 
des opérations de régulation ou des centres de recherche. Cette ouverture internationale est très fructueuse et améliore, dans chaque pays, la qualité du débat local.

On constate, dans tous les pays, le souci de respecter le rôle des élus locaux : les forums ouverts permettent l'échange, mais n'ont pas de légitimité pour décider. Les élus doivent être impliqués très tôt et ont ainsi la possibilité d'organiser les débats sur l'évaluation des projets. Certains pays donnent un veto formel aux collectivités locales (Finlande, Suisse), mais il est souvent informel, comme en Suède ou en France.

Notons quelques facteurs positifs qui favorisent le débat : du temps et quelques moyens; l'utilisation d'outils variés pour l'interaction dont le «face to face », l'organisation de groupes «panel », des conférences de consensus, des enquêtes larges. La démarche par étapes favorise l'interaction, comme l'étude d'alternatives quand elles sont de réelles alternatives.

Il faut noter plusieurs obstacles : il vaut mieux en tenir compte pour ne pas trop en être victime. Ainsi, la focalisation des débats autour de choix énergétiques du futur (venant aussi bien des pro comme d'antinucléaires) gêne la discussion sur la gestion des déchets : on doit souvent rappeller qu'il faut gérer les déchets quel que soit le futur du nucléaire. Une démarche centrée sur la technique, la tradition du secret sont des obstacles bien connus : c'est un changement de culture des organisations nécessairement lent, qu'il faut réussir. L'ouverture internationale en est un des moyens.

Enfin, dans les débats souvent difficiles, on voit certains transférer leur refus des déchets sur l'organisation qui en a la charge, devenue un bouc émissaire : ainsi l'ANDRA doit-elle défendre sa rigueur professionnelle contre des critiques radicales. Il faut refuser ces critiques déplacées.

\section{Conclusion}

Gérer les déchets radioactifs n'est pas une tâche de tout repos. Mais dans la mesure où nous savons respecter les processus dans lesquels nous sommes impliqués, si nous acceptons les règles d'un dialogue respectueux des différents publics, si la mobilisation d'un potentiel de recherche diversifié et de qualité se maintient, nous pourrons avancer. En sachant que nous traitons, pour la première fois, les impacts de l'activité humaine à très long terme, au-delà des prochaines glaciations : nous sommes en avance dans le sens du développement durable. 


\section{Pour en savoir plus}

ANDRA (2002) Où sont les déchets radioactifs en France ?, rapport de l'Observatoire national de l'ANDRA, ANDRA, Collection «Les Références ».

Barré B., Foos J., Le Bars Y., Marignac Y., Klapish R. (2002) Déchets nucléaires : comment les gérer?, AFAS Sciences, pp. 14-23, n²002-2.

Barthe Y. (2000) La mise en politique des déchets nucléaires, l'action publique aux prises avec les irréversibilités techniques, thèse.

Bataille C. (1993) Mission de médiation sur l'implantation de laboraloires de recherche souterrains, rapport au Premier ministre. La Documentation française, Paris, 1994.

Bataille C. (1996) L'évolution de la recherche sur la gestion des déchets nucléaires à haute activité, Tome I : les déchets civils, AN 2689/Sénat 299, 1996.

Bataille C. (2001) Les possibilités d'entreposage à long terme de combustibles nucléaires irradiés, AN 3101/Sénal 347, 2001

INRA-Europe (2002) Eurobarometer 56.2, European and Radioactive waste report. DG Press and Comunication Europen Commission.

IRSN (2001) Perception des risques et de la sécurité, résultats du sondage de novembre 2001.

IRSN (2002) Perception des risques et de la sécurité, résultats du sondage de novembre 2002.

Le Bars Y. (2000), «Pour un inventaire national de référence des déchets radioactifs», La Documentation Française, Paris, 2000

Le Bars Y. (2001) Entre technique et société, les acquis de la gestion des déchets radioactifs en France, La Jaune et la Rouge, le dossier du nucléaire, pp. 57-64 (novembre 2001).

Le Bars Y. (2002) La France nucléaire, matières et sites 2002, préface, pp. V-IX. Wise, Paris, 2001.

Le Bars Y. (2002) Les processus de gestion des déchets radioactifs, Gérer les risques collectifs, Chap. 8, pp. 157-178. Éditions de l'Aube Datar, série Bibliothèque des territoircs.

Le Bars Y. (2003) Gérer les déchets radioactifs: un agenda sensible, Les nouveaux chemins de l'énergie, Chap. XIII, pp. 201-208. Alpharès, Paris.

National Research Council (2003) One step at a Time. The National Academics Press, Wahington D.C. (Y. Le Bars, reviewer).

Petit J.C. (1993) Le stockage des déchets radioactifs : perspective historique et analyse sociotechnique, thèse.

Rivasi M. (2000) Les conséquences des installations de stockage des déchets nucléaires sur la santé publique et l'environnement, AN 2257/Sćnat 272, 2000.

\section{Annexe 1}

Département de la Meuse - Arrondissement de Bar-le-Duc Syndicat intercommunal à vocation unique des pays de la Saulx et du Perthois

\section{Extrait du procès-verbal des délibérations du comité syndical du 3 juin 1994}

L'an mil neuf cent quatre-vingt-quatorze, le 3 juin, le Syndicat intercommunal à vocation unique des pays de la Saulx et du Perthois étant assemblé en session 
ordinaire, au lieu habituel de ses séances, après convocation légale, sous la présidence de M. J.F. RENARD, Président,

Monsieur le Président a ouvert la séance et a présenté le projet de Laboratoire de recherches sur la gestion des déchets radioactifs :

AU MOTIF QUE, investi d'une mission de Développement local, le SIVU ne peut pas se désintéresser d'un projet aussi important que le Laboratoire de recherches sur la gestion des déchets radioactifs,

AU MOTIF QUE, à ce titre, il a le devoir d'examiner successivement et les unes par rapport aux autres, les incidents de l'éventualité d'une localisation de ce laboratoire sur son territoire,

AU MO'TIF QUE l'intercommunalité sous-tend et sous-tendra toujours fortement les actions menées ou soutenues par le SIVU,

AU MOTIF QUE l'aménagement du territoire ne peut pas être une démarche à sens unique et qu'ainsi les secteurs du Territoire qui ont des potentialités à mettre au service de la Nation ont le devoir de le faire,

AU MOTIF QUE le débat sur le nucléaire ne doit pas être confondu avec le traitement de ses effets,

CONSIDÉRANT que la cristallisation des volontés collectives s'est faite autour d'une volonté première de mettre en valeur les capacités d'accueil touristique de notre petite région,

CONSIDÉRANT qu'à aucun endroit où des activités nucléaires se sont installées, il n'a pu être prouvé que la fréquentation touristique ait eu à en souffrir : la vallée de la Loire, près de la centrale de Chinon, le lac du Der, entre la base aérienne de Saint-Dizier, qui renferme des armes nucléaires, et le centre de stockage de l'Aube (et il y a bien d'autres exemples),

CONSIDÉRANT que, par contre, de telles activités s'accompagnent de la venue de personnes ou personnalités de haut renom culturel et scientifique,

CONSIDÉRANT que de telles localisations véhiculent avec elles des aménagements qui permettent d'évaluer et de garantir la sécurité et la qualité de l'information relative au risque nucléaire,

CONSIDÉRANT qu'il est par ailleurs indispensable que de telles installations $\mathrm{s}$ 'insèrent dans un environnement de qualité exemplaire dont les pollutions de toute sorte soient absentes ou en voie de maîtrise très avancée,

CONSIDÉ RANT que notre petite région n'est pas, hélas, dans cette configuration, tant il reste à faire vis-à-vis des assainissements de villages, restauration de la qualité des cours d'au, suppression des décharges, protection des captages, mise aux normes des bâtiments d'élevage, etc.,

SOUS RÉSERVE que certains articles de la loi du 30 décembre 1991 soient précisés : l'article 2, qui laisse un ambiguïté sur la réversibilité du stockage ; l'article 4, qui autorise une décision unilatérale du Parlement sans consultation préalable, 
SOUS RÉSERVE, au cas où un stockage serait décidé, que la récupérabilité des colis stockés soit prouvée,

SOUS RÉSERVE qu'une structure de concertation soit mise en place par le SIVU et qu'elle soit ouverte à toutes les sensibilités,

SOUS RÉSERVE que la population locale soit très largement associée par l'information aux travaux de recherche qui seront menés et ce pendant toute leur durée et à leur issue,

SOUS RÉSERVE qu'un comité scientifique, intégré dans la structure de concertation et totalement indépendant de l'ANDRA, soit associé à cette information, SOUS RÉ SERVE que des moyens soient donnés au SIVU des pays de la Saulx et du Perthois pour créer sur son territoire les structures nécessaires à l'hébergement des chercheurs attachés au laboratoire et à l'accueil hôtelier des visiteurs,

SOUS RÉSERVE que le pôle agroalimentaire de Bar-le-Duc soit doté par des fonds publics de moyens lui permettant de faire accréditer une chaîne de contrôle de la conformité aux normes internationales admises en terme de radioactivité des aliments humains et animaux,

SOUS RÉSERVE que l'implantation du laboratoire soit réfléchie de sorte à préserver le paysage qui l'environne et n'apparaisse pas comme une construction totalement atypique dans sa région d'accueil,

SOUS RÉSERVE qu'à l'issue des travaux de construction, des mesures d'accompagnement soient prévues pour éviter l'effet néfaste qu'aurait un arrêt brutal des travaux, c'est-à-dire l'application de la procédure « Grands chantiers »,

Le Comité syndical s'étant exprimé par un vote public, à bulletin secret, par :

35 voix « pour », 9 voix « contre », 1 bulletin nul, 1 abstention,

\section{DÉCIDE, sous réserve de ce qui a été dit plus haut}

1. d'accepter que soit étudiée la faisabilité de l'implantation sur son territoire d'un laboratoire de recherche en vue d'un stockage souterrain RÉVERSIBLE des déchets radioactifs à vie longue, dans les conditions de fonctionnement et avec les réserves décrites plus haut;

2. de mutualiser les ressources qui en découleraient entre TOUTES les communes adhérentes du SIVU ;

3. de réserver majoritairement ces ressources à l'augmentation de l'accompagnement financier réglementaire des mesures de toute nature visant à une meilleure maîtrise de la qualité de l'environnement.

Fait et délibéré en séance les jour, mois et an ci-dessus

Pour copie conforme,

Le Président, J.F. RENARD 


\section{Annexe 2}

\section{Pour une éthique des relations de l'ANDRA avec ses publics}

\section{Charte des relations de l'ANDRA avec ses publics}

\section{Préambule}

La gestion des déchets radioactifs pose des questions où se mêlent des aspects techniques et sociaux. Elle doit intégrer les impacts potentiels à long terme, et mobiliser un ensemble de connaissances complexes. Elle fait intervenir divers acteurs, sociaux et économiques, et suscite des interrogations, éventuellement certains rejets, engendre des inquiétudes, voire des peurs.

Les propositions de gestion s'alimentent de recherches scientifiques et techniques mais doivent intégrer d'autres dimensions, où une place significative est attribuée à la réflexion sur la protection des générations futures - dans une perspective de développement durable (au sens de la conférence de Rio, 1992).

L'ANDRA, en tant qu'établissement public indépendant créé par la loi du 30 dêcembre 1991, est l'opérateur chargé, par les pouvoirs publics, d'une mission de gestion industrielle à long terme de tous les types de déchets radioactifs, d'une mission de recherche pour la définition de solutions nouvelles, et d'une mission d'information.

L'objectif de cette charte est de définir les principes qui inspireront les relations de l'ANDRA avec ses partenaires et ses publics, permettant interactions et apprentissage mutuel.

\section{Principe général}

L'ANDRA agit dans le cadre fixé par la loi de 1991 et le contrat quadriennal, signé en juillet 2001 avec ses ministères de tutelle. Ce contrat souligne en particulier que la mission d'information 'doit « répondre aux exigences d'information et de transparence vis-à-vis du public en matière de déchets radioactifs et de leur gestion » et consiste d'abord à «proposer une information claire et vérifiable sur les déchets radioactifs ».

L'ANDRA doit rendre compte de son fonctionnement, apporter son expertise, s'appliquer à comprendre et intégrer les préoccupations de ses partenaires. Parmi ceux-ci, une attention particulière est portée aux élus et aux Commissions locales d'information (CLI), à proximité de ses implantations. Pour autant, les populations locales, ou plus éloignées des sites, ne sont pas écartées, pas plus que les acteurs impliqués dans le déroulement des travaux et des recherches, les médias et tous les 
partenaires désireux d'acquérir des connaissances ou d'obtenir des explications sur les opérations effectuées, leur suivi, afin d'émettre des opinions.

Ce principe général se décline selon quatre engagements.

\section{Écoute}

L'ANDRA prête la plus grande attention aux opinions des interlocuteurs qui observent son activité. Elle participe aux débats, conférences, panels où il lui est demandé de témoigner, dans le respect des règles démocratiques. Elle favorise des échanges réguliers avec les diverses «parties prenantes» de sa mission de gestion des déchets radioactifs, dans un souci de protection de l'environnement et de la santé publique.

\section{Échanges}

L'Agence répond aux questions qui relèvent de sa compétence et de son savoirfaire. Si l'ANDRA rend compte de ses activités aux autorités de tutelle et de contrôle, elle communique au public des informations à la demande de celui-ci, avec clarté et précision. Elle s'implique activement dans l'information sur la gestion des déchets radioactifs en France, ne se substituant pas aux autres acteurs, mais en veillant à respecter les divers modes de débats.

\section{Rigueur}

L'ANDRA fonde sa légitimité sur le caractère rigoureux qui gouverne sa politique scientifique et sa politique industrielle, dans le respect de son strict rôle et de celui de ses partenaires. Les documents qu'elle fournit sont produits à un instant donné, donc évolutifs, et étayent sa participation aux débats scientifiques, ainsi qu'à ceux qui touchent aux problèmes sociétaux.

\section{Sincérité}

L'exigence de sincérité, dans l'information et les explications diffusées par l'Agence, suppose de ne jamais induire le public en erreur, volontairement ou par omission consciente, en particulier pour ce qui concerne la protection de l'homme et de l'environnement. L'Agence met à la disposition du public les éléments utiles à la compréhension de son activité, traduisant les faits dont elle dispose à un moment donné et précisé, en s'efforçant de rendre son propos accessible.

le 25 septembre 2002

Yves Le Bars

Président du Conseil d'administration
François Jacq

Directeur général 